\title{
Genetic determinants controlling maize rubisco activase gene expression and a comparison with rice counterparts
}

\author{
Yu Zhang, Yong Zhou, Qian Sun, Dexiang Deng, Huanhuan Liu, Saihua Chen and Zhitong Yin * (D)
}

\begin{abstract}
Background: Rubisco activase (RCA) regulates the activity of Rubisco and is a key enzyme of photosynthesis. RCA expression was widely reported to affect plant photosynthesis and crop yield, but the molecular basis of natural variation in RCA expression in a wide range of maize materials has not been fully elucidated.

Results: In this study, correlation analysis in approximately 200 maize inbred lines revealed a significantly positive correlation between the expression of maize RCA gene $Z m R C A \beta$ and grain yield. A genome-wide association study revealed both cis-expression quantitative trait loci (cis-eQTLs) and trans-eQTLs underlying the expression of ZmRCA $\beta$, with the latter playing a more important role. Further allele mining and genetic transformation analysis showed that a 2-bp insertion and a 14-bp insertion in the promoter of ZmRCA conferred increased gene expression. Because rice is reported to have higher RCA gene expression than does maize, we subsequently compared the genetic factors underlying RCA gene expression between maize and rice. The promoter activity of the rice RCA gene was shown to be stronger than that of the maize RCA gene, suggesting that replacing the maize RCA gene promoter with that of the rice RCA gene would improve the expression of RCA in maize.
\end{abstract}

Conclusion: Our results revealed two DNA polymorphisms regulating maize RCA gene ZmRCA $\beta$ expression, and the RCA gene promoter activity of rice was stronger than that of maize. This work increased understanding of the genetic mechanism that underlies RCA gene expression and identify new targets for both genetic engineering and selection for maize yield improvement.

Keywords: Rubisco activase (RCA), Photosynthesis, Maize, Rice, Quantitative trait locus (eQTL), Promoter

\section{Background}

Ribulose-1,5-bisphosphate carboxylase/oxygenase (Rubisco) catalyzes the first step of photosynthetic carbon assimilation and is a rate-limiting enzyme of photosynthesis. In higher plants, Rubisco activity is regulated by a second enzyme named Rubisco activase (RCA) [1-6]. RCA removes inhibitory sugar phosphates from the active sites of Rubisco in an ATP-dependent way and thus activates Rubisco [7]. The endogenous levels of RCA have been widely reported to affect plant photosynthesis and crop yield. In rice, RCA plays an important role in the regulation of non-steady-state

\footnotetext{
* Correspondence: ztyin@yzu.edu.cn

Jiangsu Key Laboratory of Crop Genetics and Physiology/Co-Innovation Center for Modern Production Technology of Grain Crops/Key Laboratory of Plant Functional Genomics of the Ministry of Education/Jiangsu Key Laboratory of Crop Genomics and Molecular Breeding, Yangzhou University, Yangzhou 225009, China
}

photosynthesis [8]. Overexpression of the RCA gene improves rice grain yield, whereas anti-sense expression shows the opposite effect $[9,10]$. In wheat, RCA expression showed a significantly positive linear correlation with plant productivity [11]. In soybean, the expression levels of two RCA genes showed significant correlations with photosynthesis and grain yield [12, 13]. In maize, high-yielding population showed higher RCA expression [14, 15], and the RCA expression displayed significant and positive correlations with grain yield in a wide range of maize inbred lines [16].

Characterization of the genetic elements regulating RCA gene expression could facilitate the modulation of the RCA gene for improving crop yield. For this purpose, genetic determinants that control the expression of RCA genes have been identified in various crops. Functional analysis of the rice RCA gene promoter revealed several cis-elements that regulate its expression [17]. 
Expression quantitative trait locus (eQTL) mapping identified several eQTLs that control the expression of soybean RCA genes $[12,13]$. A screen of a leaf cDNA library using a yeast one-hybrid $(\mathrm{Y} 1 \mathrm{H})$ system identified two bZIP transcription factors that interact with the promoter of the soybean RCA gene [18]. Maize is one of the most important $\mathrm{C}_{4}$ crops worldwide. Although $\mathrm{C}_{4}$ monocot plants including maize show higher photosynthetic efficiency due to an extra biochemical $\mathrm{CO}_{2}$-concentrating mechanism (CCM) yielding higher $\mathrm{CO}_{2}$ partial pressure $\left(p \mathrm{CO}_{2}\right)$ at Rubisco catalytic sites, their Rubisco activation status is generally lower than that of $\mathrm{C}_{3}$ monocot plants [19]. Therefore, there should be much room to increase maize Rubisco activation state and subsequently photosynthesis. Because of the primary role of RCA to activate Rubisco in vivo, mapping the genetic factors underlying RCA expression in maize is of paramount importance.

Recently, based on linkage analysis using a maize recombinant inbred line (RIL) population derived from two parental inbred lines, we identified several eQTLs underlying RCA gene expression [20]. Although these eQTLs can help to understand the regulation of the RCA gene in maize, the molecular basis of natural variation in RCA expression in a wide range of maize materials has not been fully elucidated. Genome-wide association analysis (GWAS) based on linkage disequilibrium (LD) studies in a natural population allows for evaluation of multiple alleles underlying the natural variation of a target trait. Additionally, GWAS can increase resolution and facilitate gene discovery [21].

Rice, one of the most important $C_{3}$ crops, shows a higher level of RCA gene expression than that of maize [22]. The difference in RCA gene expression between maize and rice most likely required the evolution of a different regulatory mechanism. Therefore, the higher level of RCA gene expression in rice than that in maize suggests that rice might have stronger RCA gene regulatory factors, in the form of either cis- or trans-regulatory factors, than maize. To date, no information is available on the similarities or differences in the RCA gene regulatory elements between maize and rice. If rice is confirmed to have a stronger RCA regulator than maize, future high-yield breeding of maize can focus on replacing its weaker RCA gene regulator with the stronger one from rice.

In maize, two RCA genes, $Z m R C A \alpha$ and $Z m R C A \beta$, have been identified and cloned. The $Z m R C A \beta$ expression level was approximately 10 -fold that of $Z m R C A \alpha$. Additionally, compared with $Z m R C A \alpha, Z m R C A \beta$ showed a much higher correlation with grain yield [16]. In most studies, only one RCA gene, OsRCA, has been identified in the rice genome [23]. Alternative splicing of this gene produces two RCA isoforms, the short $\beta$ form and the long $\alpha$ form [24]. In this study, GWAS was employed to map eQTLs for the maize RCA gene, $Z m R C A \beta$, and the rice RCA gene, OsRCA. Subsequently, sequencing of target DNA segments and candidate gene association were employed to screen for elite alleles of the RCA gene promoter in these two species. Finally, genetic transformation experiments were conducted to examine the differences in RCA gene promoter activity between maize and rice. Our findings showed that a 2bp insertion and a 14-bp insertion in the promoter of $Z m R C A \beta$ conferred increased gene expression and that the rice RCA gene promoter had higher activity than that of the maize RCA gene promoter.

\section{Results}

\section{Variation of $Z m R C A \beta$ expression and its correlation with grain yield}

Because alternative splicing of the $Z m R C A \beta$ precursor RNA creates two transcripts [16], we measured the transcript expression of this gene by designing primers that detect the two transcripts simultaneously (Additional file 1 : Table S1). The transcript expression of $Z m R C A \beta$ and grain yield exhibited wide phenotypic variations in the maize association populations (Table 1 and Fig. 1). The means, standard deviations, ranges, skewness, broad-sense heritability values, and analysis of variance (ANOVA) for $Z m R C A \beta$ transcript expression and grain yield are presented in Table 1. The average relative value of $Z m R C A \beta$ transcript expression were 1.46 and 1.38 in 2015 and 2016 growing season, respectively. The variation range of $Z m R C A \beta$ expression was larger, 95\% of lines fell in the range of $0.21-3.13$ in 2015 and $0.17-2.93$ in 2016. The average of grain yield were 45.60 and $45.11 \mathrm{~g}$ per plant $(\mathrm{g} /$ plant) in 2015 and 2016 growing season, respectively. The variation range of grain yield was larger, $95 \%$ of lines fell in the range of 19.83-71.37 g/plant in 2015 and 17.32-

Table 1 Descriptive statistics, ANOVA and broad-sense heritability for RCA gene expression and grain yield

\begin{tabular}{lllllll}
\hline Trait & Mean \pm SD & Range & Skew & $\mathrm{G}^{\mathrm{a}}$ & $\mathrm{R}^{\mathrm{b}}$ & $H^{2}(\%)^{\mathrm{c}}$ \\
\hline ZmRCAß-2015 & $1.46 \pm 0.85$ & $0.09-5.66$ & 2.01 & $\mathrm{~s}$ & $\mathrm{~ns}$ & 0.75 \\
ZmRCA -2016 & $1.38 \pm 0.79$ & $0.10-4.92$ & 1.90 & $\mathrm{~s}$ & $\mathrm{~ns}$ & 0.80 \\
ZmYield-2015 & $45.60 \pm 13.15$ & $11.00-83.95$ & 0.10 & $\mathrm{~s}$ & $\mathrm{~ns}$ & 0.86 \\
ZmYield-2016 & $45.11 \pm 14.18$ & $13.38-93.78$ & 0.53 & $\mathrm{~s}$ & $\mathrm{~ns}$ & 0.76 \\
OsRCA-2016 & $0.94 \pm 0.54$ & $0.20-2.55$ & 1.15 & $\mathrm{~s}$ & $\mathrm{~ns}$ & 0.64 \\
\hline
\end{tabular}

Note: The $Z m R C A \beta$ and $O s R C A$ expression data are represented by the relative values compared to their reference genes, ZmActin and OsActin, respectively.

The unit for grain yield is gram per plant (g/plant). $Z m R C A \beta$ expression measured in 2015 and 2016 is termed as ZmRCA -2015 and ZmRCA -2016, respectively. Maize grain yield measured in 2015 and 2016 is termed as ZmYield-2015 and ZmYield-2016, respectively. OsRCA expression measured in 2016 is termed as OsRCA-2016

s: significant difference at $P<0.01$; ns: not significant

a Genotype;

${ }^{\mathrm{b}}$ Replication;

c Broad-sense heritability 


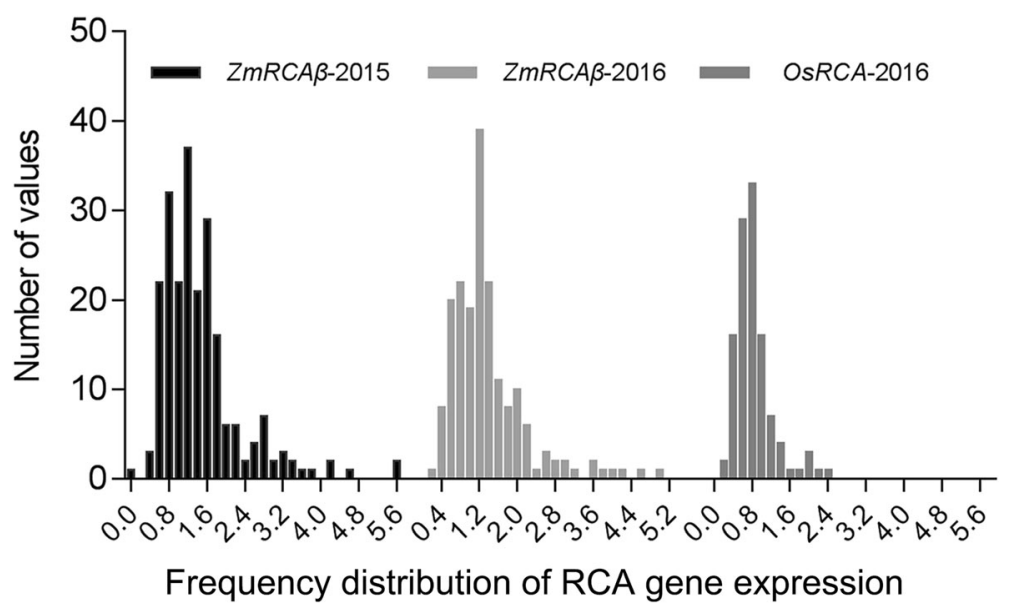

Fig. 1 Frequency distribution of the expression of $Z m R C A \beta$ and $O s R C A$

$72.90 \mathrm{~g} /$ plant in 2016. ANOVA indicated that $Z m R C A \beta$ transcript expression and grain yield were significantly affected by genotype $(P<0.01)$. The heritability $\left(H^{2}\right)$ estimates for $Z m R C A \beta$ transcript expression ranged from 75 to $80 \%$, and for grain yield from 76 to $86 \%$ (Table 1). Overall, the transcript expression of $Z m R C A \beta$ and grain yield clearly exhibited wide natural variation in the maize association populations.

In our previous study, we determined the transcript and protein expressions of $Z m R C A \beta$ and a newly discovered RCA gene $Z m R C A \alpha$ in 123 maize inbred lines. The transcript expression of $Z m R C A \beta$ was observed to correlate well with its protein expression; both the transcript and protein expressions of $Z m R C A \beta$ were significantly correlated with grain yield, and more importantly, the correlations were much higher than those between $Z m R C A \alpha$ and grain yield [16]. In the current study, we focused on $Z m R C A \beta$ transcript expression, and observed that during the two growing seasons of 2015 and 2016, the $Z m R C A \beta$ transcript expression also displayed a significant correlation with the corresponding grain yield $(P<0.01)$. Pearson phenotypic correlation coefficient between $Z m R C A \beta$ transcript expression and grain yield was 0.38 in the 2015 growing season and 0.36 in the 2016 growing season (Fig. 2). The above results indicate that the $Z m R C A \beta$ transcript expression could affect maize grain yield.

\section{Genome-wide association study for $Z m R C A \beta$ expression}

To identify the genetic loci associated with $Z m R C A \beta$ expression, GWAS was performed by using 558,629 genomic single nucleotide polymorphisms (SNP) and the $Z m R C A \beta$ transcript expression data generated in an association population comprising 222 and 182 maize lines in 2015 and 2016, respectively (see Plant materials and methods). To avoid spurious associations, such as the historical relationships and selection patterns of the maize lines in the association population, multiple statistical models were evaluated in the GWAS. Visual observation of quantile-quantile (Q-Q) plots provides an indication of the accuracy of the model used to analyze the data. As shown in the Q-Q plots (Fig. 3A, C), the Pvalues of the mixed linear model (MLM) were close to the expected values, indicating this model was suitable for reducing the effects of population structure on $Z m R C A \beta$ transcript expression. Thus, we conducted
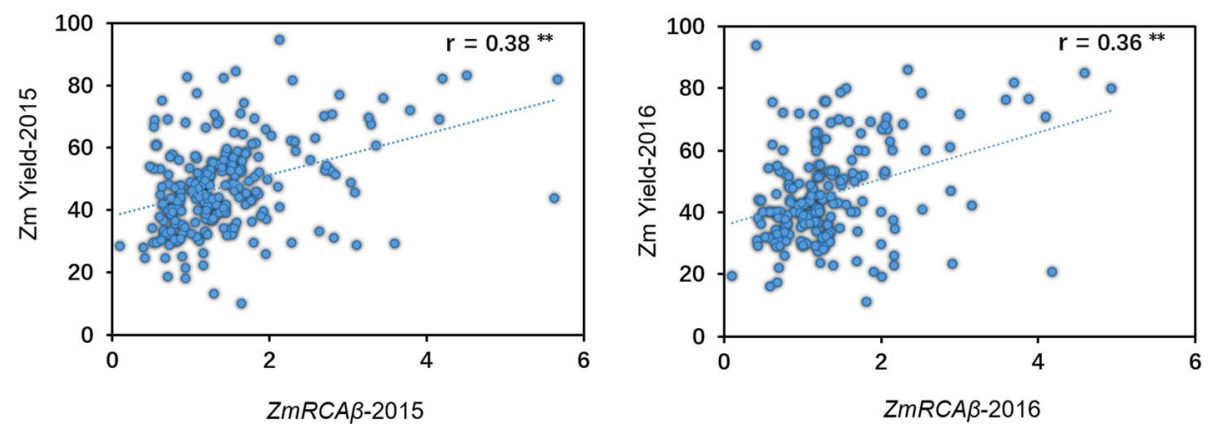

Fig. 2 Correlation analysis for the transcript expression of $Z m R C A \beta$ and the grain yield in the association population. $* *$ significant at $P<0.01$ 
A

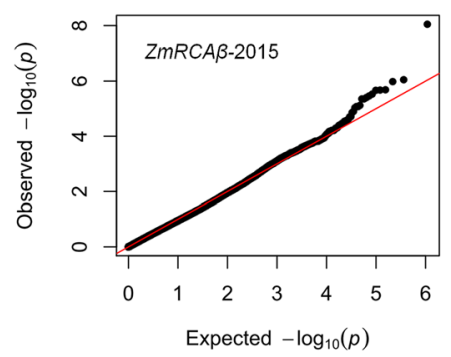

C

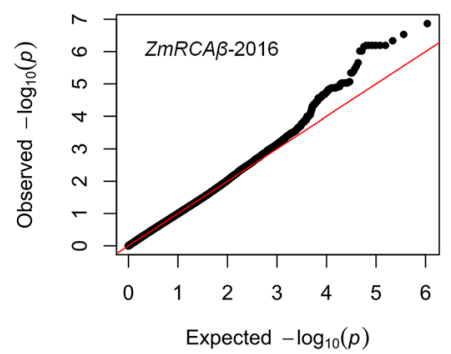

B

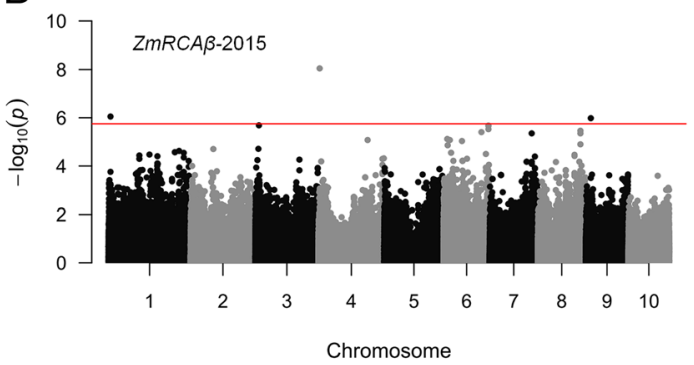

D

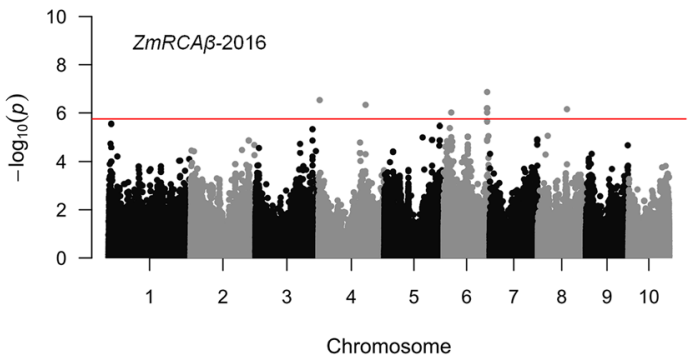

Fig. 3 GWAS for the expression of ZmRCAß. a, c Quantile-quantile plot for the GWAS under a mixed linear model (MLM). b, d Manhattan plot for the GWAS. Note: The red line indicates a significant association signal $(-\log P>5.75)$

GWAS for ZmRCAß transcript expression with the MLM model.

Three and 12 SNPs were identified with significant marker-trait associations with $Z m R C A \beta$ transcript expression at the Bonferroni-adjusted significance threshold in 2015 and 2016 growing seasons, respectively (Table 2 and Fig. 3B, D). These SNPs were distributed on chromosomes $1,4,6,8$, and 9 , and the phenotypic variation explained by each SNP ranged from 13.65 to $29.71 \%$. Interestingly,
GWAS for grain yield showed that the significant marker chr6.S-28129864 for ZmRCA 3 expression (Table 2) was also significantly associated with grain yield (Additional file 9: Table S6). Of the significant marker-trait associations for $Z m R C A \beta$ expression, the marker PZE-104005992 was repeatedly detected in 2015 and 2016 growing seasons. A survey of the maize reference genome sequence (https://www. maizegdb.org/) revealed that this marker was located at 3.6 $\mathrm{Mb}$ upstream of $Z m R C A \beta$ on chromosome 4.

Table 2 SNP markers associated with the expression of ZmRCAß in maize association population

\begin{tabular}{|c|c|c|c|c|c|}
\hline Trait & Marker & Chromosome & Position & $-\log P$ & $R^{2}(\%)$ \\
\hline \multirow[t]{3}{*}{ ZmRCAß-2015 } & PZE-104005992 & 4 & $4,289,239$ & 8.05 & 24.87 \\
\hline & chr1.S_7,076,785 & 1 & $7,076,785$ & 6.05 & 13.98 \\
\hline & chr9.S_18,890,334 & 9 & $18,890,334$ & 5.98 & 13.65 \\
\hline \multirow[t]{12}{*}{$Z m R C A \beta-2016$} & chr6.S_159,773,778 & 6 & $159,773,778$ & 6.87 & 20.31 \\
\hline & PZE-104005992 & 4 & $4,289,239$ & 6.53 & 29.71 \\
\hline & chr4.S_172,763,634 & 4 & $172,763,634$ & 6.34 & 18.74 \\
\hline & chr6.S_159,772,882 & 6 & $159,772,882$ & 6.19 & 17.93 \\
\hline & chr6.S_159,772,884 & 6 & $159,772,884$ & 6.19 & 17.93 \\
\hline & chr6.S_159,772,951 & 6 & $159,772,951$ & 6.19 & 17.93 \\
\hline & chr6.S_159,773,131 & 6 & $159,773,131$ & 6.19 & 17.93 \\
\hline & chr6.S_159,773,245 & 6 & $159,773,245$ & 6.19 & 17.93 \\
\hline & chr6.S_159,773,246 & 6 & $159,773,246$ & 6.19 & 17.93 \\
\hline & chr8.S_107,014,893 & 8 & $107,014,893$ & 6.16 & 17.65 \\
\hline & chr6.S_28,129,864 & 6 & $28,129,864$ & 6.02 & 17.26 \\
\hline & chr6.S_159,772,707 & 6 & $159,772,707$ & 6.02 & 17.75 \\
\hline
\end{tabular}


Candidate gene association analysis of the polymorphisms in the promoter region of $Z m R C A \beta$ The 1700-bp region of the $Z m R C A \beta$ promoter, which was previously shown to be sufficient for the proper regulation of expression in plants [25, 26], was cloned and sequenced in the maize association population. The promoter sequences of 186 inbred lines of different geographic origins were successfully cloned and sequenced. Multiple sequence alignment of the promoter region revealed 79 polymorphic sites in the 186 maize inbred lines (Additional file 2: Table S2). To determine whether these sequence polymorphisms were related to the observed variation in $Z m R C A \beta$ transcript expression, candidate-gene association analysis was performed. Two polymorphisms, a 2-bp indel and 14-bp indel, were repeatedly detected to be significantly associated with the expression of $Z m R C A \beta$ in both 2015 and 2016 growing seasons at $P<0.01$ (Table 3 ). The 2-bp indel located 144-bp upstream of the start codon of $Z m R C A \beta$ explained 4.24 and $6.15 \%$ of the phenotypic variation in 2015 and 2016, respectively. The 14-bp indel located 210-bp upstream of the start codon of $Z m R C A \beta$ explained 3.80 and $5.98 \%$ of the phenotypic variation in 2015 and 2016, respectively.

\section{Haplotype analysis for ZmRCA $\beta$ promoter}

Based on the polymorphisms significantly related with RCA expression, the promoter sequence of $Z m R C A \beta$ was divided into 3 haplotypes, ZmHap1, ZmHap2 and ZmHap3 (Fig. 4A). The inbred lines harboring each of these haplotypes are shown in Additional file 3: Table S3. The inbred lines carrying the ZmHap1 showed the highest $Z m R C A \beta$ expression and the highest grain yield, and those carrying the ZmHap3 showed the lowest $Z m R C A \beta$ expression and the lowest grain yield (Fig. 4B, C).

A dual-luciferase reporter system was used to verify the ZmHap1 promoter of $Z m R C A \beta$ had higher activity than that of the ZmHap3 promoter. We cloned ZmHap1 and ZmHap3 promoters from inbred lines CIMBL57 and CIMBL88, respectively. These two inbred lines showed the highest and lowest levels of $Z m R C A \beta$ expression, respectively. The ZmHap1 or ZmHap3 promoter-driven LUC (ZmHap1 or ZmHap3 pro-LUC) and CaMV35S promoter-driven REN (35S-REN; as an internal control) were constructed in the same plasmid (Fig. 5A), and expressed

Table 3 Polymorphisms in the $Z m R C A \beta$ promoter region significantly associated with $Z m R C A \beta$ expression in maize association population

\begin{tabular}{lccccc}
\hline $\begin{array}{l}\text { Polymorphic } \\
\text { type }\end{array}$ & Position & $\begin{array}{l}-\log P \\
2015\end{array}$ & 2016 & 2015 & 2016 \\
\hline 2-bp indel & -114 & 2.32 & 3.13 & 4.24 & 6.15 \\
14-bp indel & -210 & 2.12 & 3.05 & 3.80 & 5.98 \\
\hline
\end{tabular}

Significant association: $-\log P>2$ in maize protoplasts, rice protoplasts, and tobacco (Nicotiana benthamiana) leaves. The dual luciferase expression system with no promoter to drive LUC was used as a negative control (Fig. 5A). The LUC/REN ratio, which reflects in vivo promoter activity, was monitored. The LUC/ REN value of ZmHap1 promoter was significantly higher than that of the ZmHap3 promoter in maize protoplasts, rice protoplasts, and the leaves of tobacco (Fig. 5B, C). We also compared the activity of ZmHap1 and ZmHap3 promoters using rice callus genetic transformation experiment, and observed a similar result (Additional file 4: Figure S1). These results confirmed that the ZmHap1 promoter had higher activity than that of the ZmHap3 promoter. Therefore, ZmHap1 can be regarded as an elite allele for the $Z m R C A \beta$ promoter.

\section{Comparison of RCA gene regulatory elements between maize and rice}

To compare the regulating strength of RCA gene expression determinants between maize and rice, the transcript expression of rice RCA gene OsRCA was determined in an association population containing 114 cultivars with extensive genetic diversity, and the genetic determinants underlying the transcript expression of OsRCA were identified using the same method employed in maize as described above. Similar to the measurement of $Z m R C A \beta$ expression, because alternative splicing of the OsRCA precursor RNA produces two transcripts [24], we also measured the transcript expression of this gene by designing primers that detect the two transcripts simultaneously (Additional file 1: Table S1). The transcript expression of OsRCA exhibited wide natural variations in the rice association population (Table 1 and Fig. 1). A 12.75-fold difference occurred among the cultivars in the rice association population (Table 1). GWAS using the rice association population revealed 5 SNPs significantly associated with OsRCA transcript expression (Additional file 5: Table S4 and Additional file 6: Figure S2).

The promoter sequence of 1500-bp was cloned and sequenced in 107 rice cultivars. Candidate-gene association analysis revealed 12 polymorphisms significantly associated with OsRCA transcript expression in its promoter region (Additional file 7: Table S5). Based on these polymorphisms, the OsRCA promoter was divided into 5 haplotypes (Additional file 3: Table S3). The cultivars carrying OsHap1 had the highest OsRCA expression and those carrying OsHap5 had the lowest OsRCA expression (Additional file 8: Figure S3B).

The aforementioned experiments of protoplast transient expression, leaf transient expression, and callus genetic transformation were conducted to compare the promoter activity of maize $Z m R C A \beta$ and rice OsRCA. We cloned OsHap1 and OsHap5 promoters from rice cultivars Jing 185-7 and Saber, respectively. These two cultivars showed 
A

\begin{tabular}{|c|c|c|c|c|}
\hline \multirow{2}{*}{ Haplotype } & \multicolumn{2}{|c|}{ Position } & \multicolumn{2}{c|}{ number of maize inbred lines } \\
\cline { 2 - 5 } & $\mathbf{- 2 1 0}$ & $\mathbf{- 1 4 4}$ & $\mathbf{2 0 1 5}$ & $\mathbf{2 0 1 6}$ \\
\hline ZmHap1 & $14 \mathrm{bp}$ & $2 \mathrm{bp}$ & 73 & $71^{*}$ \\
\hline ZmHap2 & 0 & $2 \mathrm{bp}$ & 85 & $83^{*}$ \\
\hline ZmHap3 & 0 & 0 & 28 & 28 \\
\hline
\end{tabular}

$\mathrm{B}$

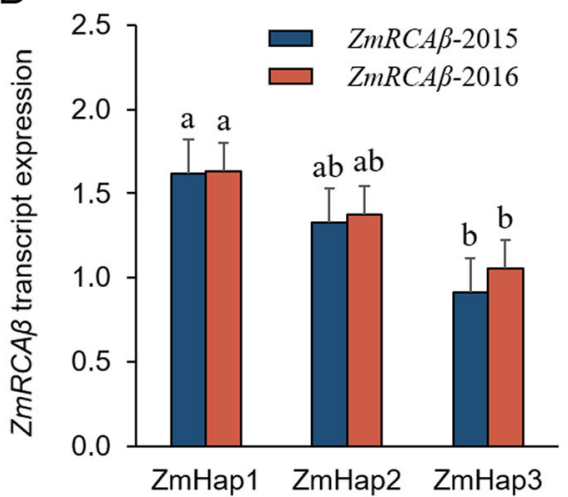

C

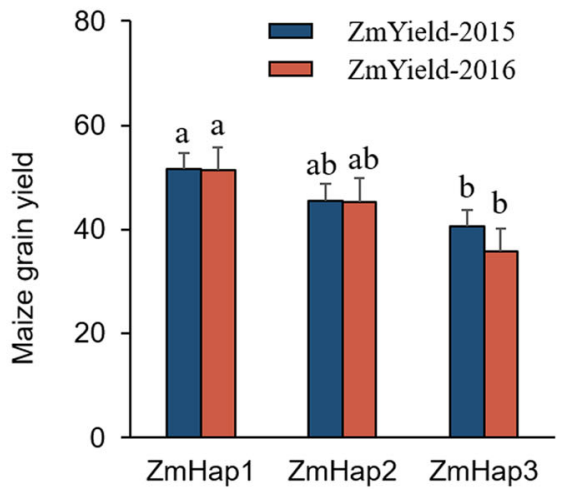

Fig. $4 Z m R C A \beta$ promoter haplotypes and the average $Z m R C A \beta$ expression and grain yield for each haplotype. a Haplotype analysis of the $Z m R C A \beta$ promoter regions. The dark gray-shaded cells represent the favorable alleles. *Two lines harboring ZmHap1 and two lines harboring ZmHap2 used for determining ZmRCA expression and grain yield were not available in 2016. b. $\mathbf{c}$ The average expression of ZmRCA 3 and maize grain yield for different haplotypes. Error bars represent the standard error. The number of data points used for calculating the standard error is the product of number of maize lines belonging to each haplotype and biological replicates of each line. Multiple comparison of phenotypic data was performed using Fisher's protected Least Significant Difference (LSD) test. Different English alphabet means significant difference at $P<0.05$

the highest and lowest levels of OsRCA expression, respectively, in the rice association population. Each of the OsHap1 and OsHap5 promoter sequences was fused with the LUC or GUS reporter gene in plasmid (Fig. 5A and Additional file 4: Figure S1A). In the protoplast and leaf transient expression experiments, the LUC/REN value of OsHap1 was significantly higher than that of OsHap5, confirming that the OsHap1 promoter had higher activity than that of the OsHap5 promoter (Fig. 5B, C). The LUC/REN value of rice stronger OsHap1 promoter was significantly higher than maize stronger ZmHap1 promoter. Notably, the LUC/REN value of the rice weaker OsHap5 promoter was even higher than the maize stronger ZmHap1 promoter although the difference observed in maize protoplast experiment was not significant (Fig. 5B, C). The similar results were also observed in the callus genetic transformation experiment (Additional file 4: Figure S1B). These results suggested that the activity of the rice RCA promoter was higher than that of the maize RCA promoter.

\section{Discussion}

Mapping of favorable alleles underlying $\operatorname{ZmRCA} \beta$ expression for maize high-yield breeding

Strong evidence exists that two traits are causally related when the two traits are significantly correlated with one another and have coincident QTLs that are consistent with the correlation [27]. In the present study, the expression level of $Z m R C A \beta$ was positively correlated with grain yield in an association population consisting of approximately 200 maize inbred lines. Furthermore, GWAS for grain yield showed that one QTL for grain yield coincided with the eQTL for $Z m R C A \beta$ expression (Additional file 9: Table S6). We also observed similar results in our previous study using 123 maize inbred lines [16]. These data suggest that the RCA gene might be a potential factor affecting plant productivity. Therefore, identifying the key genomic regions that regulate the expression level of $Z m R C A \beta$ could aid in maize high-yield breeding via the marker-assisted-selection (MAS) method.

The success of MAS breeding depends on the gene mapping resolution and germplasm diversity. In our previous study, we detected five eQTLs for $Z m R C A \beta$ in a RIL population containing 242 maize lines [20]. However, these eQTLs were mapped to relatively large mapping intervals, and no causal explicit genetic elements could be inferred for regulating ZmRCA $\beta$ expression. Additionally, these eQTLs do not represent the elite alleles underlying natural variation of $Z m R C A \beta$ expression because they were mapped in a bi-parental 


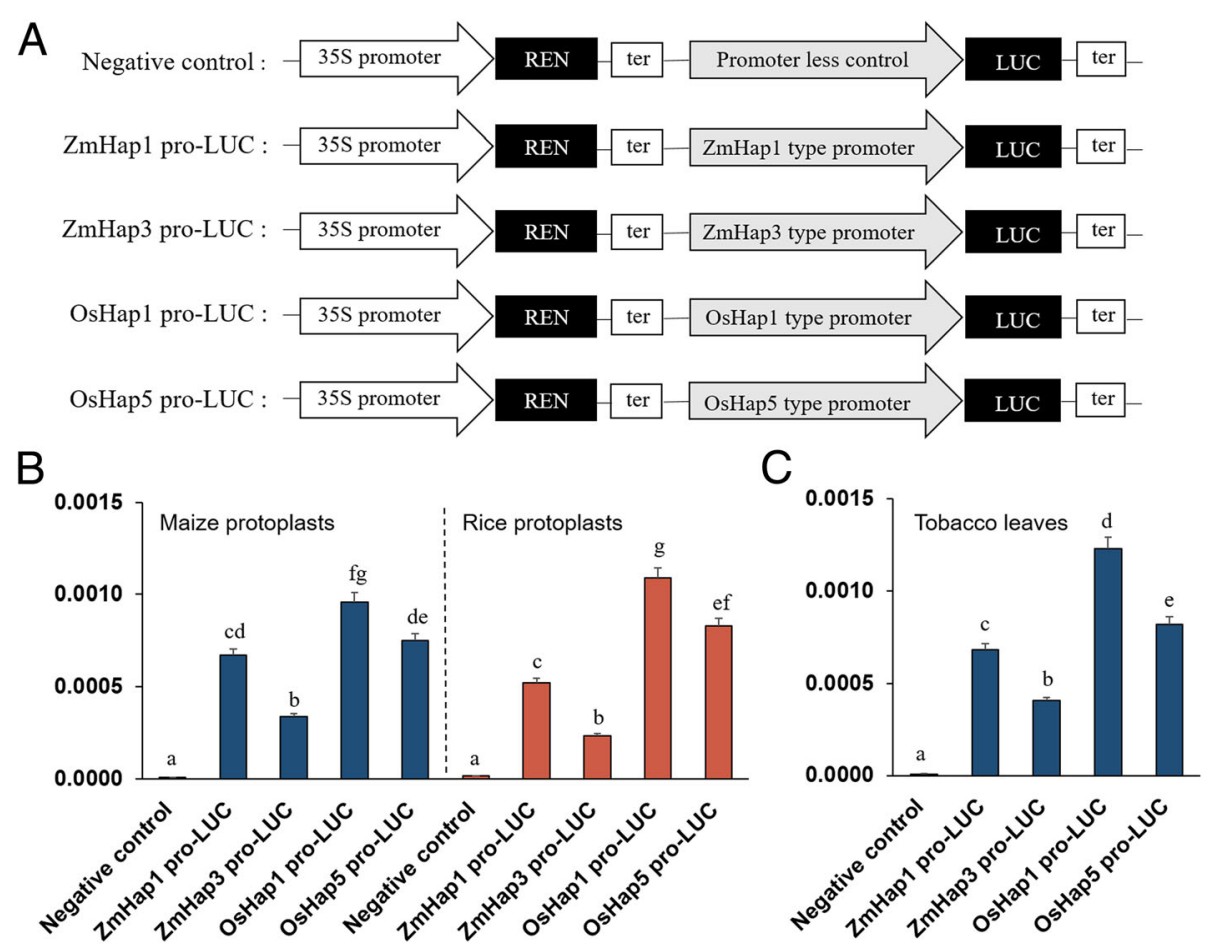

Fig. 5 Activity of different types of RCA promoters. a Schematic diagrams of the dual luciferase assay vector. REN, Renilla luciferase; LUC, firefly luciferase; ter, terminator. $\mathbf{b}$ Ratios of LUC and REN activity in maize and rice protoplasts transformed with recombinant plasmids containing different types of ZmRCA and OSRCA promoters and negative control. c Ratios of LUC and REN activity in tobacco (Nicotiana benthamiana) leaves transformed with recombinant plasmids containing different types of ZmRCA and OsRCA promoters and negative control. Multiple comparison of phenotypic data was performed using LSD test. Different English alphabet means significant difference at $P<0.05$

population. GWAS combined with target region association analysis in a diverse association population can provide the opportunity to discover elite alleles of interest and delimit them into a relatively small genomic region [28]. This method takes advantage of current and historical recombination events for gene fine mapping, particularly in maize, a species that shows high diversity and rapid LD decay. Therefore, in the present study, GWAS combined with target region association analysis was employed to discover favorable alleles underlying $Z m R C A \beta$ expression in a maize association population. The results show that the favorable alleles underlying $Z m R C A \beta$ expression were finely mapped.

In the present study, GWAS initially detected one major eQTL, PZE-104005992, on chromosome 4 in approximately 200 inbred lines with extensive genetic diversity. This eQTL was repeatedly detected across two field-growth conditions and explained a large phenotypic variation (Table 2). We also detected this eQTL in our previous study [20]. Therefore, PZE-104005992 can be considered as a primary breeding target for modulation of $Z m R C A \beta$ expression. Subsequently, we focused on refined mapping of this eQTL. Considering that the expression of one gene is usually affected by the sequence variation in its promoter and that the genomic location of the $Z m R C A \beta$ promoter coincided with the eQTL PZE-104005992, we employed candidate-gene association analysis to the $Z m R C A \beta$ promoter. The results show that two polymorphic sites, a 2-bp indel and a 14bp indel, in the promoter were significantly associated with gene expression (Table 3). The inbred lines carrying the 2-bp and 14-bp insertion showed higher $Z m R C A \beta$ expression and higher yields than those of other inbred lines (Fig. 4). Finally, genetic transformation experiments showed the promoter with the 2-bp and 14-bp insertion had higher activity than that of other promoters (Fig. 5 and Additional file 4: Figure S1). Based on these results, we conclude that the promoter carrying the 14-bp and 2-bp insertion might be a favorable allele for maize high-yield breeding. Functional markers are derived from polymorphic sites within genes that are causally involved in phenotypic trait variation [29]. The 2-bp and 14-bp indels identified in the present study can be used for development of functional markers.

\section{Genetic mechanism underlying RCA gene expression}

When an eQTL is within $5 \mathrm{Mb}$ of its target gene, a ciseQTL is defined; otherwise, the eQTL is a trans-eQTL [30-33]. The identification of a cis-eQTL suggests that the genetic determinants for gene expression are 
adjacent to or coincident with the genomic region of the target gene. A trans-eQTL indicates that genetic determinants far from the target gene control its expression [34]. In the present study, GWAS revealed 14 and 5 SNPs that were significantly associated with the expression of $Z m R C A \beta$ and OsRCA, respectively (Table 2 and Additional file 5: Table S4). Of the 14 significant SNPs for $Z m R C A \beta$, the marker PZE-104005992 was $3.6 \mathrm{Mb}$ from $Z m R C A \beta$ in the maize reference genome (https:// www.maizegdb.org/) and was called a cis-eQTL, whereas the other SNPs were trans-eQTLs. Of the 5 significant SNPs for OsRCA, the marker id 11011480 was $0.7 \mathrm{Mb}$ from $O s R C A$ in the rice reference genome (http://www. ricedata.cn/gene/) and was a cis-eQTL, whereas the other SNPs were trans-eQTL. Notably, the cis-eQTL showed a much stronger association with gene expression and explained a much larger proportion of phenotypic variation than any individual trans-eQTL in both rice and maize (Table 2 and Additional file 5: Table S4). We also obtained similar results in our previous study: in maize, we identified 1 cis-eQTL and 4 trans-eQTLs for the expression of $Z m R C A \beta$ in a RIL population under two field-growth environments and observed that the cis-eQTL explained the most phenotypic variation and was stably expressed across the different environments [20]. In soybean, we identified 13 SNPs significantly associated with the expression of the RCA gene GmRCA $\beta$ and found that the cis-eQTL had a stronger association with gene expression than that of the transeQTLs [12]. These results indicate that the expression of the RCA gene might be controlled by a combination of both cis-acting and trans-acting eQTLs and that the ciseQTL played a dominant role in regulating RCA gene expression.

Promoters can affect the action of both cis- and transeQTLs on the expression of a target gene via alterations in its cis-acting regulatory elements or transcription factor binding sites [34]. Therefore, DNA sequence variation in promoters is a potential factor explaining the diversity of $Z m R C A \beta$ and OsRCA expression. In the present study, candidate-gene association analysis revealed 2 polymorphic sites in the $Z m R C A \beta$ promoter and 12 polymorphic sites in the OsRCA promoter that were significantly associated with gene expression (Table 3 and Additional file 7: Table S5). The RCA gene expression was significantly different in haplotypes of maize inbred lines or rice cultivars divided according to the detected significant association polymorphic sites (Fig. 4 and Additional file 8: Figure S3). Furthermore, genetic transformation experiments confirmed that the two types of promoters of $Z m R C A \beta$ and OsRCA had different activity (Fig. 5 and Additional file 4: Figure S1). These results suggest that promoter sequence variation can influence RCA gene expression. A similar result was also observed in soybean in which sequence variation in the $G m R C A \beta$ promoter contributed to diversity in GmRCA $\beta$ expression [12].

\section{Comparison of the promoter activity between maize and rice provides a potential approach for maize high- photosynthetic-efficiency breeding}

In the present study, we used qRT-PCR to determine maize and rice RCA gene expression. Although gene expression can be measured quickly and accurately, this method provides only the relative expression data of the targeted gene compared with a reference gene. Because the absolute values of reference gene expression are not available in maize and rice, we could not compare the RCA gene expression magnitude between these two species. Therefore, we focused on comparing the promoter activity of maize $Z m R C A \beta$ and rice OsRCA using genetic transformation experiments.

Different results have been reported on the cell typespecific expression pattern of maize RCA [35-37]. A high density of immune-gold particles for RCA was observed to be located in chloroplast stroma of both bundle sheath (BS) and mesophyll (M) cells in maize leaf [35]. Similarly, although the authors did not point out, an immunoblot analysis of BS and M cells total proteins in maize revealed that RCA accumulated to a similar level in the two types of cells (according to Fig. 7B in the paper by Wostrikoff et al. [36]). In contrast, maize RCA was shown to express at a much higher level in BS cells than in $\mathrm{M}$ cells as revealed by large-scale quantitative proteomics [37] and RNA-seq analysis [38]. If the promoter of ZmRCA $\beta$ and OsRCA has cell type-specific expression pattern between BS and M cells, it should show a different expression magnitude between maize and rice leaf tissues that contain a different ratio of $\mathrm{M}$ and BS cell number. In the present study, neither the promoter activity of a maize haplotype, either ZmHap1 or ZmHap3, nor the promoter activity of a rice haplotype, either OsHap1 or OsHap5, showed significantly different activity between maize and rice protoplasts (Fig. $5 \mathrm{~B}$ ). These results suggest that the $Z m R C A \beta$ and OsRCA promoter did not yield significant cell type-specific activity in BS and $\mathrm{M}$ cells.

Genetic transformation experiments revealed that the activity of the rice OsRCA promoter was significantly higher than that of the maize $Z m R C A \beta$ promoter (Fig. 5 and Additional file 4: Figure S1). This result is consistent with the finding that the RCA gene in maize showed a lower expression level than that in rice [22]. Compared with maize, rice Rubisco operates in a much lower $\mathrm{CO}_{2}$ environment and at a higher activation state [19]. To maintain the high activation state of Rubisco, the rice RCA gene promoter likely evolved a high regulating strength to keep the RCA gene expression at a high 
level. Considering $\mathrm{C}_{4}$ plants have an extra $\mathrm{CCM}$ and consequently much higher level of $\mathrm{CO}_{2}$ concentration at their Rubisco catalytic sites than $\mathrm{C}_{3}$ plants, we attempted to search for the difference in the RCA gene promoter between $C_{3}$ and $C_{4}$ species. A survey of the PlantCARE database [39] showed that, compared with $\mathrm{C}_{4}$ species including maize, the promoter from $C_{3}$ species including rice have a significantly higher number of cis-acting elements associated with light regulation (Additional file 10: Table S7). Because the RCA gene is highly regulated by light [40], the increase in number of light regulation-related cis-elements might lead to an RCA gene promoter with increased activity. Future studies are required to compare the regulating strength of the RCA promoter between $C_{3}$ and $C_{4}$ plants using multiple $C_{3}$ and $C_{4}$ species.

As it was shown that the activity of the rice OsRCA promoter was higher than that of the maize $Z m R C A \beta$ promoter (Fig. 5 and Additional file 4: Figure S1), we suppose that maize photosynthesis could be improved by replacing the maize $Z m R C A \beta$ promoter with the rice $O s R C A$ promoter. This replacement can combine the high $\mathrm{CO}_{2}$ environment at the Rubisco catalytic sites with a high RCA gene expression in maize plants. A concern for this replacement is that it might affect the action of some heat-stress related regulatory elements that control maize RCA gene expression. RCA is known to be particularly susceptible to heat stress $[41,42]$, and it was reported that heat stress induced a new RCA isoform with higher molecular weight in maize [43]. Another concern is that the replacement might lead to a decrease of maize Rubisco content. Recently, it was reported that RCA expression negatively affected Rubisco content in transgenic rice [6]. Further studies are required to investigate the effect of the replacement on maize RCA gene expression, Rubisco content, photosynthesis, and grain yield under both common and heat-stressed conditions.

\section{Conclusion}

This study of eQTL mapping and genetic transformations of promoter regions were performed to characterize the genetic factors underlying the expression of the $Z m R C A \beta$ gene in maize. Furthermore, we compared the regulating strength of the identified factor between the $\mathrm{C}_{4}$-plant maize and the $\mathrm{C}_{3}$-plant rice. Our study showed that the RCA gene might play a role in maize grain yield. A 2-bp insertion and a 14-bp insertion in the promoter conferred higher expression of maize RCA gene. The promoter activity of the rice RCA gene was stronger than that of the maize RCA gene. The two identified indels associated with maize RCA gene can be used in marker-assisted selection breeding for high photosynthetic efficiency. More importantly, people usually transfer $\mathrm{C}_{4}$-specific genes from $\mathrm{C}_{4}$-crop maize to $\mathrm{C}_{3^{-}}$- crop rice in traditional high-photosynthetic-efficiency breeding. Our study suggested a possible and novel method in high-photosynthetic-efficiency breeding, which is, in the other way around, transferring gene from $\mathrm{C}_{3}$-crop rice to $\mathrm{C}_{4}$-crop maize.

\section{Methods}

\section{Plant materials and plant growth conditions}

A panel of 437 maize lines described previously [44] was used. All 437 lines were genotyped for SNP markers using high-throughput genotyping platforms, and approximately 558,629 polymorphisms with minor allele frequencies (MAF) $\geq 0.05$ were available for a GWAS [44]. The 437 lines were planted in two growing seasons in 2015 and 2016 on the experimental farm of the Agricultural College of Yangzhou University. In each growing season, the 437 lines were planted in a randomized block design with two replicates. Each line was planted in one row per block. The length of each row was $2.5 \mathrm{~m}$, the spacing between plants in each row was $0.25 \mathrm{~m}$, and the spacing between rows was $0.55 \mathrm{~m}$. Leaf tissues for gene expression measurement were prepared according to our previous study [16]. At $32 \mathrm{~d}$ after anthesis, the leaves located nearest to the ear were harvested from three randomly selected plants of each line per block in the morning (9:00-11:30 a.m.) on a sunny day, frozen immediately in liquid nitrogen, and stored at $-80^{\circ} \mathrm{C}$ before measuring $Z m R C A \beta$ expression. To minimize the impacts of differences in plant growth stage on gene expression measurements, we chose 222 and 182 lines with similar anthesis time in 2015 and 2016, respectively. The origin of the selected lines is shown in Additional file 3: Table S3.

A panel of 218 rice cultivars was used to evaluate OsRCA gene expression. All 218 cultivars were genotyped for SNP markers using high-throughput genotyping platforms, and approximately 36,900 polymorphisms with MAF $\geq 0.05$ were available for GWAS [45]. All cultivars were planted on the experimental farm of the Agricultural College of Yangzhou University in 2016. The cultivars were planted in a randomized block design with two replicates. Each cultivar was planted in one row per block. Row length was $1.5 \mathrm{~m}$, row distance was $0.2 \mathrm{~m}$, and plant distance in each row was $0.132 \mathrm{~m}$. At the late filling stage of development, the rice flag leaves were collected individually from three randomly selected plants of each cultivar per block in the morning (9:0011:30 a.m.) on a sunny day, frozen immediately in liquid nitrogen, and stored at $-80^{\circ} \mathrm{C}$ until further use. To minimize the impacts of differences in growth stage on the measurements of gene expression, we chose $114 \mathrm{cul}-$ tivars with similar anthesis time. The origin of the selected cultivars is shown in Additional file 3: Table S3. 
Trait measurement and phenotypic data collection

Total RNA was extracted using TRIzol reagent (Vazyme, China). We used HiScript reverse transcriptase (Vazyme, China) to prepare cDNA. Gene expression levels were determined by quantitative real-time PCR (qRT-PCR) using an SYBR Premix Ex Taq kit (Vazyme, China) on a Bio-Rad CFX96 system (Applied Biosystems). The expression of endogenous reference genes ZmActin (GenBank accession number J01238) and OsActin (GenBank accession number AB047313) was used to normalize the transcript levels in each maize and rice sample, respectively. Gene specific primers (Additional file 1: Table S1) were used for qRT-PCR. The following formula was used to obtain the normalized expression of target gene for each sample: $\Delta \Delta \mathrm{C}_{\mathrm{T}}=\left(\mathrm{C}_{\mathrm{T}}\right.$, Target $-\mathrm{C}_{\mathrm{T}}$, Actin $)$ genotype - $\left(\mathrm{C}_{\mathrm{T}}\right.$, Target $-\mathrm{C}_{\mathrm{T}}$, Actin $)$ calibrator. Each sample was prepared by mixing equal amounts of the collected leaves from the three plants described above for each line (cultivar) per block. Therefore, in each growing season, each maize line or rice cultivar contained two samples from two blocks.

Maize grain yield for each line per block was estimated using the average yield of five plants in the middle of each row. At maturity, the ears of the corresponding plants were hand harvested, dried to a constant mass, and threshed; and the mean grain yield per plant was recorded.

\section{Statistical analyses of phenotypes}

Descriptive statistical analysis and correlation analyses were performed using the statistical software package SPSS Statistics 17.0 for Windows (SPSS, Inc., Chicago, IL, USA). Two-tailed ANOVA was used for comparisons between blocks and genotypes. The broad-sense heritability $\left(H^{2}\right)$ for RCA gene expression or grain yield in each growing season was implemented by SAS 9.2 (SAS institute, Cray, USA) and was estimated by the following equation $H^{2}=\mathrm{V}_{\mathrm{g}} /\left(\mathrm{V}_{\mathrm{g}}+\mathrm{V}_{\mathrm{e}} / \mathrm{r}\right)$, where $\mathrm{V}_{\mathrm{g}}$ and $\mathrm{V}_{\mathrm{e}}$ represent genetic variance and error variance, respectively, and were calculated from the ANOVA as described above; $r$ is the block replicate number in field experiment. Frequency distribution analysis was performed using GraphPad Prism (version 7, GraphPad Software, San Diego, CA).

\section{Genome-wide association study}

TASSEL software 5.0 was used to perform GWAS [46]. To account for the effects of population structure on the mapping panel and genetic relatedness among panel members, the kinship matrix (K) of the association panel was calculated as previously described [47]. According to the Q-Q plots from the TASSEL 5.0 output, the MLM mode with $\mathrm{K}$ was chosen for the association mapping of $Z m R C A \beta$ and OsRCA expression. Markers were identified as significantly associated with expression of $Z m R C A \beta$ or OsRCA by comparison with the Bonferroni threshold $(P<1 / \mathrm{n},-\log P>5.75$ for $Z m R C A \beta,-\log P>$ 4.57 for OsRCA).

\section{Promoter-based association analysis}

CTAB method was employed to extract genomic DNA (gDNA) of maize and rice leaves [48]. The promoters of $Z m R C A \beta$ and OsRCA were amplified using specific primers (Additional file 1: Table S1) and the PCR procedure as described in our previous study [20]. The sample PCR products were then purified and sequenced by BGI (Shanghai, China). Tested sequences were aligned using CLUSTALX version 1.83 [49]. The identification of DNA variations among these genotypes and the candidate-gene association analysis between polymorphisms and phenotypes were performed using Tassel 5.0 [46]. In the candidate-gene association analysis for the RCA gene promoter, all polymorphisms with a MAF $\geq 0.05$ were considered [44].

\section{Promoter activity analysis in rice callus}

Maize and rice RCA promoters, which are $1700 \mathrm{bp}$ and $1500 \mathrm{bp}$ in length, respectively, were amplified from genomic DNA with specific primers (Additional file 1: Table S1) added with the cutting sites of HindIII and Sa1I enzymes. The amplified fragment was separated on 1\% agarose gels and purified with a gel extraction kit (Axygen) according to the manufacturer's protocol. The purified fragment was fused with the GUS reporter gene in the binary vector pCAMBIA1381Z digested with HindIII and Sa1I to construct recombinant GUS vector. Promoter-less vector pCAMBIA1381Z and CaMV 35S promoter-driving GUS vector pCAMBIA1301 were used as negative and positive controls, respectively. The recombinant GUS vector, negative control, and positive control were individually transformed into rice callus via Agrobacteriummediated transformation as described by Hiei et al. [50].

GUS activity was determined in the rice positive callus according to the method as described previously [51] with some modifications. Plant materials were immersed in $100 \mathrm{mM}$ sodium phosphate buffer ( $\mathrm{pH}$ 7.0) containing 1\% Triton X-100, 1\% DMSO, $10 \mathrm{mM}$ EDTA and $0.5 \mathrm{mg} /$ $\mathrm{ml}$ 5-bromo-4-chloro-3-indolyl glucuronide and incubated overnight at $37^{\circ} \mathrm{C}$. The staining solution was then removed, and the samples were dehydrated using $75 \%$ ethanol. GUS staining was observed under an Olympus SZX12 stereomicroscope and photographed with a digital camera (CoolSNAP, RS photometrics).

\section{Promoter activity analysis using dual luciferase assay}

A dual luciferase assay vector pGreenII0800-LUC was used to analyze the activity of the target promoter. This vector contains firefly luciferase (LUC) reporter gene 
that can be driven by the target promoter and Renilla luciferase (REN) reporter gene driven by 35S. The purified DNA fragment of the target promoter was fused with LUC reporter gene in the vector digested with HindIII and SalI enzymes to construct the recombinant vector. The vector pGreenII0800-LUC without promoter insertion before LUC reporter gene was used as negative control. The recombinant and negative control vectors were individually transformed into maize and rice protoplasts or tobacco (Nicotiana benthamiana) leaves. In the protoplast transient expression experiments, the isolation of protoplasts from maize or rice green leaves, PEG-calcium transfection of plasmid DNA, and protoplast culture were performed according to standard protocols [52]. In the leaf transient expression experiment, tobacco plants were grown until at least six leaves were available. The transfection of plasmid DNA into tobacco leaves by infiltration with Agrobacterium and transient expression assay followed standard protocols [53].

The ratio of LUC and REN activity (LUC/REN) was used to reflect the activity of the target promoter. The LUC/REN value was determined using the dual luciferase reporter assay system (Promega). Briefly, the transformed protoplasts or homogenate prepared from transformed tobacco leaves were centrifuged at $12000 \times \mathrm{g}$ for $15 \mathrm{~s}$ at room temperature, and the supernatant was removed. Next, $100 \mu \mathrm{l}$ of passive lysis buffer was added for further homogenization. Twenty microliters of lysate was mixed with $100 \mu \mathrm{l}$ of LAR II, and then the LUC activity was measured using a GloMax 20/20 luminometer (Promega). Finally, $100 \mu \mathrm{l}$ of Top \& Glo reagent was added to the reaction, and the REN activity was measured.

\section{Additional files}

Additional file 1: Table S1. Primer pairs used in this study. (DOCX 108 $\mathrm{kb})$

Additional file 2: Table S2. Polymorphic sites in the promoter region of ZmRCAB and OsRCA. (XLSX $9 \mathrm{~kb}$ )

Additional file 3: Table S3. Origin and haplotype of plant materials of maize and rice association populations in this study. (XLSX $11 \mathrm{~kb}$ )

Additional file 4: Figure S1. Activity of different types of RCA promoters. (A) Schematic diagrams of the GUS vector. GUS, $\beta$ glucuronidase; ter, terminator. (B) Histochemical staining of rice callus transformed with recombinant plasmids containing different types of $Z m R C A \beta$ and OsRCA promoters, positive control, and negative control. The result showed that GUS expression driven by the ZmHap1 promoter was stronger than that by ZmHap3 promoter, GUS expression driven by the OsHap1 promoter was stronger than that by the OsHap5 promoter, and the GUS expression driven by rice promoters was stronger than that by the maize promoters. (DOCX $14 \mathrm{~kb}$ )

Additional file 5: Table S4. SNP markers associated with the expression of OsRCA in association population. (XLSX $9 \mathrm{~kb}$ )

Additional file 6: Figure S2. GWAS for the expression of OsRCA. (A) Quantile-quantile plot for the GWAS under a mixed linear model (MLM).
(B) Manhattan plot for the GWAS. The red line indicates a significant association signal $(-\log P>4.57)$. (DOCX $248 \mathrm{~kb})$

Additional file 7: Table S5. Polymorphisms in the OsRCA promoter region significantly associated with gene expression. (XLSX $10 \mathrm{~kb}$ )

Additional file 8: Figure S3. OsRCA promoter haplotypes and the average OsRCA expression for each haplotype. (A) Haplotype analysis of the OsRCA promoter regions. The dark gray-shaded cells represent the favorable alleles. (B) The average expression of OsRCA for different haplotypes. Error bars represent the standard error. The number of data points used for calculating the standard error is the product of number of rice cultivars belonging to each haplotype and biological replicates of each cultivar. Multiple comparison of phenotypic data was performed using LSD test. Different English alphabet means significant difference at $P<0.05$. (DOCX $878 \mathrm{~kb}$ )

Additional file 9: Table S6. SNP markers associated with the maize grain yield in the association population. (XLSX $36 \mathrm{~kb}$ )

Additional file 10: Table S7. Cis-acting elements in the RCA promoter region of $C_{3}$ and $C_{4}$ species. (XLSX $12 \mathrm{~kb}$ )

\section{Abbreviations}

ANOVA: analysis of variance; $\mathrm{CCM}$ : $\mathrm{CO}_{2}$-concentrating mechanism; eQTL: Expression quantitative trait locus; GUS: $\beta$-glucuronidase; GWAS: Genome-wide association analysis; $H^{2}$ : the broad-sense heritability; indel: insertion-deletion; LD: linkage disequilibrium; LUC: firefly luciferase; MAF: minor allele frequencies; MLM: the mixed linear model; $p \mathrm{CO}_{2}$ : $\mathrm{CO}_{2}$ partial pressure; PEG: polyethylene glyco; RCA: Rubisco activase; REN: renilla luciferase; RIL: recombinant inbred line; Rubisco: Ribulose-1,5-bisphosphate carboxylase/oxygenase; SNP: single nucleotide polymorphisms

\section{Acknowledgements}

Not applicable.

\section{Authors' contributions}

YZ1 (Yu Zhang), ZY, and SC designed the research. YZ1, QS, YZ2 (Yong Zhou), DD, and $\mathrm{HL}$ contributed to sample preparation, experimental execution and data analysis. YZ1 and ZY wrote the manuscript. All authors read and approved the final manuscript.

\section{Funding}

This work received grant support in part from the National Natural Science Foundation of China (31571669), the National Transgenic Research Project of China (2018ZX08009-04B), the Jiangsu Key Research and Development Program (BE2017365), Jiangsu Agriculture Science and Technology Innovation Fund (CX (18)1001), the Jiangsu Major Variety Breeding Project (PZCZ201710), the Yangzhou University Outstanding Young Teacher Training Project, and the Priority Academic Program Development of Jiangsu Higher Education Institutions (PAPD). The funding agencies provided only the experimental cost and publication fee for this work. However, the experimental design and data collection and analysis were managed by the contributing authors.

Availability of data and materials

All data generated or analysed during this study are included in this published article (and its supplementary information files).

Ethics approval and consent to participate

Not applicable.

Consent for publication

Not applicable.

Competing interests Not applicable. 
Received: 24 March 2019 Accepted: 8 August 2019 Published online: 14 August 2019

\section{References}

1. Salvucci ME, Ogren WL. The mechanism of rubisco activase: insights from studies of the properties and structure of the enzyme. Photosynth Res. 1996;47(1):1-11.

2. Streusand VJ, Portis AR. Rubisco Activase mediates ATP-dependent activation of ribulose bisphosphate carboxylase. Plant Physiol. 1987;85(1):152-4.

3. Portis J. Regulation of ribulose 1,5-bisphosphate carboxylase/oxygenase activity. Annu Rev Plant Biol. 1992;43(1):415-37.

4. Somerville CR, Portis AR, Ogren WL. A mutant of Arabidopsis thaliana which lacks activation of RuBP carboxylase in vivo. Plant Physiol. 1982;70(2):381.

5. Bracher A, Whitney SM, Hartl FU, Hayer-Hartl M. Biogenesis and metabolic maintenance of rubisco. Annu Rev Plant Biol. 2017;68:29-60.

6. Fukayama H, Mizumoto A, Ueguchi C, Katsunuma J, Morita R, Sasayama D, et al. Expression level of rubisco activase negatively correlates with rubisco content in transgenic rice. Photosynth Res. 2018;137(3):465-74.

7. Portis AR. Rubisco activase - Rubisco's catalytic chaperone. Photosynth Res. 2003;75(1):11-27.

8. Yamori W, Masumoto C, Fukayama H, Makino A. Rubisco activase is a key regulator of non-steady-state photosynthesis at any leaf temperature and, to a lesser extent, of steady-state photosynthesis at high temperature. Plant J. 2012;71(6):871-80.

9. Wu HR, Li LB, Jing YX, Kuang TY. Over-and anti-sense expressions of the large isoform of ribulose-1,5-bisphosphate carboxylase/oxygenase activase gene in Oryza sativa affect the photosynthetic capacity. Photosynthetica. 2007:45(2):194-201.

10. Fukayama H, Ueguchi C, Nishikawa K, Katoh N, Ishikawa C, Masumoto C, et al. Overexpression of rubisco activase decreases the photosynthetic $\mathrm{CO} 2$ assimilation rate by reducing rubisco content in rice leaves. Plant Cell Physiol. 2012;53(6):976-86.

11. Ristic Z, Momcilovic I, Bukovnik U, Prasad PV, Fu J, Deridder BP, et al. Rubisco activase and wheat productivity under heat-stress conditions. J Exp Bot. 2009;60(14):4003-14.

12. Chao M, Yin Z, Hao D, Zhang J, Song H, Ning A, et al. Variation in rubisco activase (RCAbeta) gene promoters and expression in soybean [Glycine max (L.) Merr]. J Exp Bot. 2014;65(1):47-59.

13. Yin Z, Meng F, Song H, Wang X, Xu X, Yu D. Expression quantitative trait loci analysis of two genes encoding rubisco activase in soybean. Plant Physiol. 2010;152(3):1625-37.

14. Martin M, Casano LM, Zapata JM, Guera A, del Campo EM, SchmitzLinneweber $\mathrm{C}$, et al. Role of thylakoid Ndh complex and peroxidase in the protection against photo-oxidative stress: fluorescence and enzyme activities in wild-type and ndhF-deficient tobacco. Physiol Plant. 2004;122(4): 443-52.

15. Morales A, Ortega-Delgado ML, Molina-Galán J, Jiménez ESD. Importance of rubisco activase in maize productivity based on mass selection procedure. J Exp Bot. 1999;50(335):823-9.

16. Yin Z, Zhang Z, Deng D, Chao M, Gao Q, Wang Y, et al. Characterization of rubisco activase genes in maize: an alpha-isoform gene functions alongside a beta-isoform gene. Plant Physiol. 2014;164(4):2096-106.

17. Yang Z, Lu Q, Wen X, Chen F, Lu C. Functional analysis of the rice rubisco activase promoter in transgenic Arabidopsis. Biochem Bioph Res Co. 2012; 418(3):565-70.

18. Zhang J, Du H, Chao M, Yin Z, Yang H, Li Y, et al. Identification of two bZIP transcription factors interacting with the promoter of soybean rubisco Activase gene (GmRCAalpha). Front Plant Sci. 2016;7:628.

19. Sharwood RE, Ghannoum O, Kapralov MV, Gunn LH, Whitney SM. Temperature responses of rubisco from Paniceae grasses provide opportunities for improving C3 photosynthesis. Nat Plants. 2016;2(12):16186.

20. Sun $Q$, Zhang Y, Chen B, Jia B, Zhang ZL, Cui M, et al. Expression quantitative trait loci analysis of the rubisco activase gene in maize. Photosynthetica. 2017:55(2):329-37.

21. Zhang Y, Cui M, Zhang J, Zhang L, Li C, Kan X, et al. Confirmation and fine mapping of a major QTL for aflatoxin resistance in maize using a combination of linkage and association mapping. Toxins. 2016;8(9):E258.

22. Xu J, Brautigam A, Weber AP, Zhu XG. Systems analysis of cis-regulatory motifs in $C 4$ photosynthesis genes using maize and rice leaf transcriptomic data during a process of de-etiolation. J Exp Bot. 2016;67(17):5105-17.
23. To KY, Suen DF, Chen SC. Molecular characterization of ribulose-1,5bisphosphate carboxylase/oxygenase activase in rice leaves. Planta. 1999; 209(1):66-76.

24. Zhang ZL, Komatsu S. Molecular cloning and characterization of cDNAs encoding two isoforms of ribulose-1,5-bisphosphate carboxylase/oxygenase activase in rice (Oryza sativa L.). J Biochem. 2000;128(3):383-9.

25. Liu Z, Taub CC, McClung CR. Identification of an Arabidopsis thaliana ribulose-1,5-bisphosphate carboxylase/oxygenase activase (RCA) minimal promoter regulated by light and the circadian clock. Plant Physiol. 1996; 112(1):43-51.

26. Orozco BM, Ogren WL. Localization of light-inducible and tissue-specific regions of the spinach ribulose bisphosphate carboxylase/oxygenase (rubisco) activase promoter in transgenic tobacco plants. Plant Mol Biol. 1993;23(6):1129-38.

27. Thumma BR, Naidu BP, Chandra A, Cameron DF, Bahnisch LM, Liu C. Identification of causal relationships among traits related to drought resistance in Stylosanthes scabra using QTL analysis. J Exp Bot. 2001;52(355):203-14.

28. Yan J, Warburton M, Crouch J. Association mapping for enhancing maize ( L.) genetic improvement. Crop Sci. 2011:51(2):433.

29. Andersen JR, Lübberstedt T. Functional markers in plants. Trends Plant Sci Trends Plant Sci. 2003:8(11):554-60.

30. Morley M, Molony CM, Weber TM, Devlin JL, Ewens KG, Spielman RS, et al. Genetic analysis of genome-wide variation in human gene expression. Nature. 2004;430(7001):743-7.

31. Ghazalpour A, Doss S, Zhang B, Wang S, Plaisier C, Castellanos R, et al. Integrating genetic and network analysis to characterize genes related to mouse weight. PLoS Genet. 2006;2(8):e130.

32. Gatti DM, Lu L, Williams RW, Sun W, Wright FA, Threadgill DW, et al. MicroRNA expression in the livers of inbred mice. Mutati Res-fund Mol M. 2011;714(1-2):126-33.

33. Grundberg E, Kwan T, Pastinen TM. Analysis of the impact of genetic variation on human gene expression. Methods Mol Biol. 2010;628:321-39.

34. Vuylsteke M, Van Den Daele H, Vercauteren A, Zabeau M, Kuiper M. Genetic dissection of transcriptional regulation by CDNA-AFLP. Plant J. 2006;45(3): 439-46.

35. Hong J, Wang W, Jiang D, Hu D. The immunogold localization of rubisco and its activase in chloroplasts of barley and maize leaves. Acta Photophysiologica Sinica. 2004;30(5):561.

36. Wostrikoff K, Clark A, Sato S, Clemente T, Stern D. Ectopic expression of rubisco subunits in maize mesophyll cells does not overcome barriers to cell type-specific accumulation. Plant Physiol. 2012;160(1):419-32.

37. Friso G, Majeran W, Huang M, Sun Q, van Wijk KJ. Reconstruction of metabolic pathways, protein expression, and homeostasis machineries across maize bundle sheath and mesophyll chloroplasts: large-scale quantitative proteomics using the first maize genome assembly. Plant Physiol. 2010;152(3):1219-50.

38. Li P, Ponnala L, Gandotra N, Wang L, Si Y, Tausta SL, et al. The developmental dynamics of the maize leaf transcriptome. Nat Genet. 2010; 42(12):1060-7.

39. Lescot M, Dehais P, Thijs G, Marchal K, Moreau Y, Van de Peer $Y$, et al. PlantCARE, a database of plant cis-acting regulatory elements and a portal to tools for in silico analysis of promoter sequences. Nucleic Acids Res. 2002;30(1):325-7.

40. Qu D, Song Y, Li WM, Pei XW, Wang ZX, Jia SR, et al. Isolation and characterization of the organ-specific and light-inducible promoter of the gene encoding rubisco activase in potato (Solanum tuberosum). Genet Mol Res. 2011;10(2):621-31.

41. Salvucci ME, CraftsBrandner SJ. Relationship between the heat tolerance of photosynthesis and the thermal stability of rubisco Activase in plants from contrasting thermal environments. Plant Physiol. 2004;134(4):1460-70.

42. Crafts-Brandner SJ, Salvucci ME. Rubisco activase constrains the photosynthetic potential of leaves at high temperature and $\mathrm{CO} 2$. Proc Natl Acad Sci U S A. 2000;97(24):13430-5.

43. Sánchez dJE, Medrano L, Martínezbarajas E. Rubisco activase, a possible new member of the molecular chaperone family. Biochemistry. 1995;34(9):2826-31.

44. Li H, Peng Z, Yang X, Wang W, Fu J, Wang J, Han Y, Chai Y, Guo T, Yang N. Genome-wide association study dissects the genetic architecture of oil biosynthesis in maize kernels. Nat Genet. 2013;45(1):43-50.

45. Zhao K, Tung CW, Eizenga GC, Wright MH, Ali ML, Price AH, et al. Genomewide association mapping reveals a rich genetic architecture of complex traits in Oryza sativa. Nat Commun. 2011;2:467. 
46. Bradbury PJ, Zhang Z, Kroon DE, Casstevens TM, Ramdoss Y, Buckler ES, Bishop PM. Genetics and population analysis TASSEL: software for association mapping of complex traits in diverse samples. Bioinformatics. 2008:23(19):2633-5.

47. Kumar GR, Sakthivel K, Sundaram RM, Neeraja CN, Balachandran SM, Rani NS, et al. Allele mining in crops: prospects and potentials. Biotechnol Adv. 2010;28(4):451-61.

48. Murray MG, Thompson WF. Rapid isolation of high molecular weight plant DNA. Nucleic Acids Res. 1980;8(19):4321-5.

49. Thompson JD, Gibson TJ, Plewniak F, Jeanmougin F, Higgins DG. The CLUSTAL_X windows interface: flexible strategies for multiple sequence alignment aided by quality analysis tools. Nucleic Acids Res. 1997;25(25): 4876-82.

50. Hiei Y, Ohta S, Komari T, Kumashiro T. Efficient transformation of rice (Oryza sativa L.) mediated by agrobacterium and sequence analysis of the boundaries of the T-DNA. Plant J. 1994;6(2):271-82.

51. Jefferson RA, Kavanagh TA, Bevan MW. GUS fusions: beta-glucuronidase as a sensitive and versatile gene fusion marker in higher plants. EMBO J. 1987; 6(13):3901.

52. Yoo SD, Cho YH, Sheen J. Arabidopsis mesophyll protoplasts: a versatile cell system for transient gene expression analysis. Nat Protoc. 2007;2(7):1565.

53. Zahur M, Magbool A, Irfan M, Barozai MYK, Qaiser U, Rashid B, et al. Functional analysis of cotton small heat shock protein promoter region in response to abiotic stresses in tobacco using agrobacterium-mediated transient assay. Mol Biol Rep. 2009;36(7):1915-21.

\section{Publisher's Note}

Springer Nature remains neutral with regard to jurisdictional claims in published maps and institutional affiliations.

- fast, convenient online submission

- thorough peer review by experienced researchers in your field

- rapid publication on acceptance

- support for research data, including large and complex data types

- gold Open Access which fosters wider collaboration and increased citations

- maximum visibility for your research: over $100 \mathrm{M}$ website views per year

At $\mathrm{BMC}$, research is always in progress.

Learn more biomedcentral.com/submissions 